\title{
Protective Effects of the Glutamate Antagonist MK-801 on Pyrithiamine-Induced Lesions and Amino Acid Changes in Rat Brain
}

\author{
Philip J. Langlais ${ }^{1,2,3}$ and Robert G. Mair ${ }^{4,5}$ \\ ${ }^{1}$ Psychology Service, VA Medical Center, San Diego, California 92161, ${ }^{2}$ Department of Psychology, San Diego State \\ University, San Diego, California 92182, ${ }^{3}$ Department of Neurosciences, University California San Diego, La Jolla, California \\ 92093, ${ }^{4}$ Research Service, VA Medical Center, Brockton, Massachusetts 02401, and ${ }^{5}$ Department of Psychology, \\ University of New Hampshire, Durham, New Hampshire 03824
}

An acute bout of pyrithiamine-induced thiamine deficiency (PTD) produces pathologic lesions within thalamus, mammillary body, and periventricular regions of rat brain. The biological bases for these pathologic changes and their selective distribution within the brain are unclear. The type of tissue damage observed within the thalamus of PTD rats closely resembles that observed following anoxic-ischemic insults and suggests the involvement of excitotoxic amino acids in its pathogenesis. The effects of the $\mathbf{N}$-methyl-Daspartate receptor antagonist $M K-801(3 \mathrm{mg} / \mathrm{kg}$, i. p.) on brain lesions and amino acid changes have been assessed in rats killed during the late acute stages of PTD. A marked loss of neurons within midline intralaminar nuclei and the posterior nuclear group of the thalamus were observed in the early acute stage of PTD treatment. In the late acute stage, these changes were present throughout the entire thalamus and extended caudally to the periacqueductal gray and mesencephalic tegmentum. Hemorrhagic lesions were observed only in the late acute group and were the primary lesion within the mammillary body and medial and lateral geniculates. No pathologic changes were observed in hippocampus, amygdala, and cortex. MK-801 administered during the late stages resulted in a marked attenuation of necrotic damage to thalamus and periacqueductal gray and a reduction in the number and size of hemorrhagic lesions. Significant reductions of aspartate and glutamate and increases of glycine were observed in 5 regions of thalamus, the hippocampus, hypothalamus, and mammillary bodies of both the early and late acute PTD groups. Levels of GABA and taurine in caudal areas were significantly elevated in the early acute stage but were unchanged from controls in the late acute group. These amino acid changes were reduced in the MK-801 treated late acute group. These observations suggest that NMDA receptors are involved the pathogenesis of PTD-induced brain lesions and that nuclei of the intralaminar and posterior nuclear groups are most vulnerable to PTD effects.

\footnotetext{
Received Sept. 8, 1989; revised Nov. 16, 1989; accepted Nov. 30, 1989.

This work was supported by Veterans Administration Medical Research Funds and San Diego State University. We wish to thank Munazza Malik and Paul Whalen for excellent technical assistance and David Armstrong for advice and assistance with the histopathology.

Correspondence should be addressed to Dr. Philip J. Langlais, Psychology Service (116B), Veterans Administration Medical Center, 3350 La Jolla Village Dr., San Diego, CA 92161.

Copyright (C) 1990 Society for Neuroscience $0270-6474 / 90 / 051664-11 \$ 02.00 / 0$
}

The biochemical bases for histologic lesions and their selective distribution within the brain of acutely thiamine-deficient animals remain undetermined. Decreased cerebral energy production (Aikawa et al., 1984), lactate accumulation, and decreased pH (Hakim and Pappius, 1983; Hakim, 1984; Parker et al., 1984) and decreased transketolase activity (McCandless and Schenker, 1968; Pincus and Wells, 1972; Gibson et al., 1984; Butterworth, 1986) have been suggested as possible mechanisms of thiamine deficiency-induced cell death. The type of neuronal damage observed in pyrithiamine-induced thiamine deficiency (PTD) rats closely resembles that observed following anoxia (Papp et al., 1981) and glutamate-induced excitotoxicity (Armstrong-James et al., 1988). Decreased levels of glutamate and GABA within thalamus but not other brain structures following recovery from PTD (Thompson and McGeer, 1985; Heroux and Butterworth, 1988; Langlais et al., 1988a) also suggest that these amino acids may be specifically involved in PTDinduced thalamic lesions. Seizures are prevalent in PTD animals and coincide with the onset of histological damage (Hakim and Pappius, 1983). These observations also suggest an excitotoxic mechanism in PTD since penicillin-induced focal cortical seizures produce similar thalamic lesions through excess glutamate release (Collins and Olney, 1982).

The present study examined the effects of the $N$-methyl-Daspartate (NMDA) receptor antagonist, MK-801, on late-stage PTD-induced lesions of thalamus, mammillary body, and periacqueductal gray within the right hemisphere. A separate group of PTD rats killed in an earlier stage of acute thiamine deficiency and without MK-801 treatment was also examined. Amino acid concentrations were determined in 5 regions of thalamus, the hippocampus, hypothalamus, and mammillary body of the left hemisphere of the PTD and pair-fed control groups.

The results of this study provide evidence for the involvement of NMDA receptor activation in the pathogenesis of PTD-induced lesions. Since thiamine deficiency is responsible for similar damage in Wernicke-Korsakoff's syndrome, these findings suggest an important rationale for treatment of this human neuropathologic condition. Pathologic change was first observed as a necrotic lesion in intralaminar and posterior nuclear groups of the thalamus. Hemorrhagic lesions in mammillary body, medial, and lateral geniculate nuclei appeared later in acute PTD treatment. Finally, this study demonstrates a unique temporal course and anatomic distribution of changes of aspartate, glutamate, GABA, and glycine, within affected (thalamus and mammillary bodies) and unaffected (hippocampus) brain areas. Preliminary results of this study have been presented in abstract form (Langlais et al., 1988b). 


\section{Materials and Methods}

\section{Experimental animals}

Adult, male Sprague-Dawley rats, 8 weeks old ( $280-300 \mathrm{gm})$ were used in this study. All animals were individually housed and maintained under conditions of constant temperature, humidity, and light/dark (12 $\mathrm{hr} / 12 \mathrm{hr}$ ) cycles. Animals were randomly assigned to one of the following treatment groups:

Group 1. Pair-fed controls. Rats $(N=11)$ were fed a thiamine-deficient chow (Teklad Diets, Madison, WI) and given daily injections of thiamine $(0.4 \mathrm{mg} / \mathrm{kg}$, i.p.). The amount of diet given daily was equal to the average amount of thiamine-deficient chow consumed by the PTD groups ( 2 and 3 ) on the equivalent day of treatment.

Group 2. Early acute PTD. Rats $(N=7)$ were fed the thiaminedeficient chow and given daily injections of pyrithiamine hydrobromide (0.25 mg, i. p.); (Sigma Chem. Co., St. Louis, MO). Neurological status was monitored as previously described (Mair et al., 1985), and the animals were killed approximately $24-36 \mathrm{hr}$ following loss of righting reflexes and within 1-2 hr following onset of either spontaneous or sensory evoked seizures (between day 10-11 of treatment). Loss of righting reflexes was measured by failure to land on all 4 legs when released upside down from $40 \mathrm{~cm}$ above a soft cushion.

Group 3. Late acute PTD. Rats $(N=11)$ were treated exactly as group 2 but were allowed to progress to a more severe stage of thiamine deficiency characterized by opisthotonos. Animals were killed on days 11-12, approximately $1 \mathrm{~d}$ longer than group 2 and 6-14 hr following onset of opisthotonos. Despite hourly examinations, one animal was found dead and was excluded from the remainder of the study.

Group 4. Late acute PTD $+M K-801$. Rats $(N=11)$ were treated with thiamine-deficient chow and pyrithiamine as in groups 2 and 3. In addition, the selective NMDA receptor antagonist MK-801 (Wong et al., 1986; a generous gift from Ms. Carolyn Stemmler, Merck, Sharp \& Dohme Research Laboratories, West Point, PA) was administered ( $3 \mathrm{mg} / \mathrm{kg}$. i. p.) every $8 \mathrm{hr}$ following loss of righting reflexes. To insure that MK-801 arrested and not merely delayed the pathologic process, group 4 animals were kept in acute thiamine deficiency 12-18 $\mathrm{hr}$ longer than group 3 before being killed. Two animals died during the last stage of treatment and were excluded from the study.

Group 5. Pair-fed controls $+M K-801$. Rats $(N=11)$ were treated with thiamine-deficient diet and thiamine injection as in group 1 . In addition, these animals were administered MK-801 in the same manner as group 4 animals and were killed at the same treatment period as group 4.

\section{Biochemical analyses}

Following decapitation, the brain was rapidly removed $(30-45 \mathrm{sec})$ and the left hemisphere immediately frozen on dry ice and stored at $-80^{\circ} \mathrm{C}$. Prior to dissection, the hemisphere was kept at $-20^{\circ} \mathrm{C}$ for $8-10 \mathrm{hr}$, the area of midbrain-thalamus removed, cut into $1.0-\mathrm{mm}$-thick coronal sections and placed on a $-10^{\circ} \mathrm{C}$ dissecting table (Harvard Apparatus, Newton, MA). Anterior thalamus (AT), medial thalamus (MT), lateral thalamus (LT), posterior medial thalamus (PMT), posterior lateral thalamus (PLT), hypothalamus (HY), hippocampal formation (HF), and mammillary body (MB) were dissected as shown in Figure 1 using the atlas of Paxinos and Watson (1986). A $0.1 \mathrm{~m}$ perchloric acid (PCA) extract was prepared and stored at $-80^{\circ} \mathrm{C}$ until the time of assay. Tissue amino acids were measured by treatment of the $0.1 \mathrm{M}$ PCA extract with orthopthaldialdehyde (OPA) reagent, separation of the amino acid-OPA derivatives by isocratic, reverse-phase HPLC and quantitation by electrochemical detection as previously described (Langlais et al., 1988a). The average peak heights obtained from HPLC-EC analyses of 2 separate aliquots of tissue PCA extract were used to determine amino acid concentration and are expressed as $\mathrm{nmol} / \mathrm{mg}$ protein (Lowry et al., 1951).

\section{Histological analyses}

The right hemisphere was fixed in $0.1 \mathrm{M}$ phosphate buffer, $\mathrm{pH} 7.4$, containing $2.5 \%$ glutaraldehyde $/ 4 \%$ paraformaldehyde for $24-36 \mathrm{hr}$ and subequently transferred into $10 \%$ and then $20 \%$ glycerol solutions containing $2 \%$ DMSO in $0.1 \mathrm{~m}$ phosphate buffer for storage. Frozen coronal sections, $40 \mu \mathrm{m}$ thick, were serially cut from the level of the anterior septum to the posterior pontine tegmentum. Adjacent sections were stained with either Luxol fast blue-hematoxylin-eosin or cresyl violet and examined microscopically for histopathological changes.

\begin{tabular}{|c|c|c|c|c|}
\hline \multirow[b]{2}{*}{ Symptoms/procedure } & \multicolumn{4}{|l|}{ Group } \\
\hline & Early ac & & Late acute & $\begin{array}{c}\text { Late acute } \\
+ \text { MK801 }\end{array}$ \\
\hline $\begin{array}{l}\text { Seizures duration } \\
\text { Number of animals }\end{array}$ & $\begin{array}{l}1 \\
7\end{array}$ & $-2 \mathrm{hr}$ & $\begin{array}{l}12-20 \mathrm{hr} \\
10\end{array}$ & $\begin{array}{l}\text { Absent } \\
9\end{array}$ \\
\hline $\begin{array}{l}\text { Opisthotonos duration } \\
\text { Number of animals }\end{array}$ & $\begin{array}{l}\text { Absent } \\
4\end{array}$ & $\begin{array}{l}<1 \mathrm{hr} \\
3\end{array}$ & $\begin{array}{l}6-14 \mathrm{hr} \\
10\end{array}$ & $\begin{array}{l}\text { Absent } \\
9\end{array}$ \\
\hline Day of death & & -11 & $11-12$ & $12-13$ \\
\hline
\end{tabular}

\section{Statistical analyses}

The biochemical data were examined by analysis of variance. Comparisons between means of the various treatment groups were performed using post hoc Scheffe test.

\section{Results}

\section{Behavioral observations}

Treatment with PTD resulted in aphagia and adipsia, followed by ataxia and paresis, then the loss of righting reflexes on treatment days 8-9, onset of seizures (generalized and myoclonic) on days $9-10$, and opisthotonos on days 10-11. The duration of these neurological symptoms and the time of death of animals in the 3 PTD groups are summarized in Table 1. All animals in the late acute (LA) group developed opisthotonos, whereas LA animals given MK-801 (group 4) failed to show any signs of seizure activity or opisthotonos despite an additional 12-18 hr of thiamine deficiency. Furthermore, profound sedation as evidenced by total absence of any motor activity, partially closed eyes, and respiratory depression were observed in both the pairfed control and PTD animals following MK-801 administration. These effects may have contributed to the death of 2 animals in the LA + MK-801 group.

\section{Anatomic observations}

\section{Gross observations}

Numerous petecchiae were observed on the medial surface in the regions of thalamus, mammillary body, periacqueductal gray, and floor of the third and fourth ventricles of each brain from the LA group. In addition, marked edema and tissue cavitation were evident in thalamus of most LA animals. By contrast, several animals of the LA + MK-801 group had few or no hemorrhages and little evidence of edema or necrosis on the medial surface of the brain. No gross changes were observed in any of the early acute PTD brains.

\section{Histological observations}

Early acute $(E A)$ group. All brains contained lesions within midline and medial intralaminar nuclei and posterior nuclear group of the thalamus (Fig. 2). The lesion was characterized by severe ncuronal loss and palcness of ncuropil. At rostral levels of thalamus (Fig. 2, $A-C$ ), this pattern was observed within the central medial (CM), interoanteromedial (IAM), paracentral (PC), and gelatinosus (G) nuclei. The mediodorsal nucleus was totally spared in 2 cases and a mild loss of neurons restricted to ventral MD was observed in the remaining 5 brains. At more caudal levels (Fig. 2, $D-F$ ), the lesion was observed within parafascicu- 


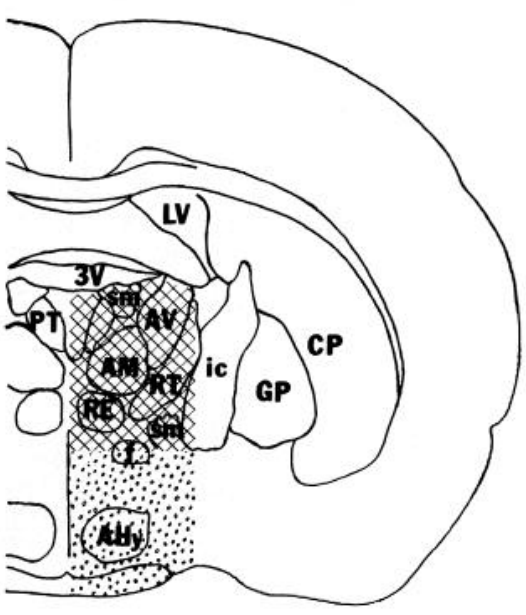

$-1.3 \mathrm{~mm}$

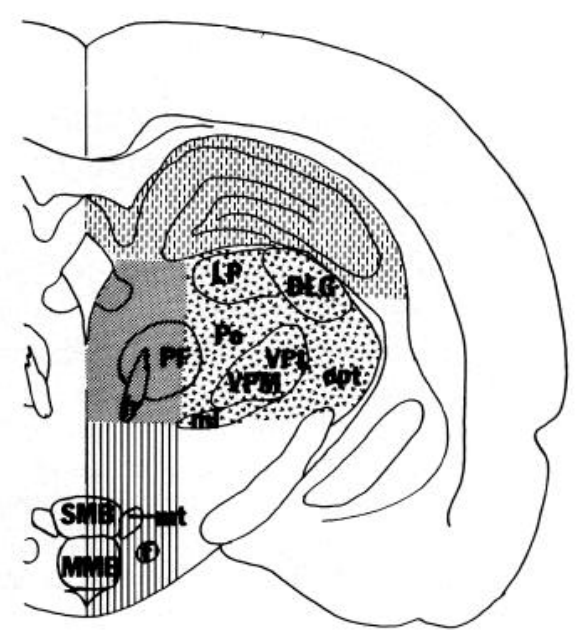

$-4.3 \mathrm{~mm}$

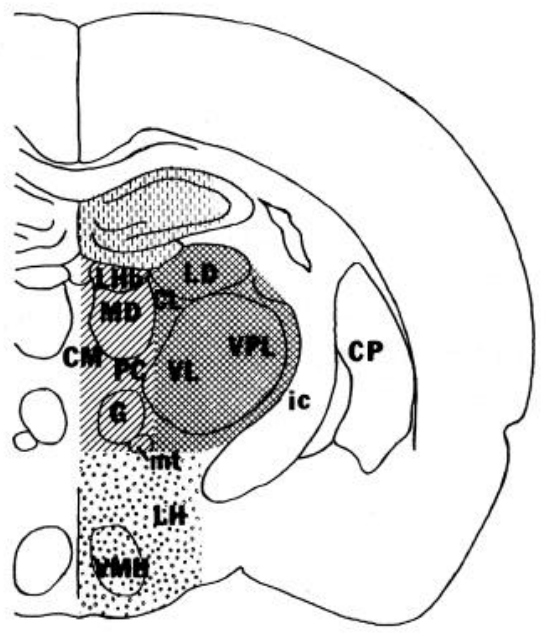

$-2.3 \mathrm{~mm}$

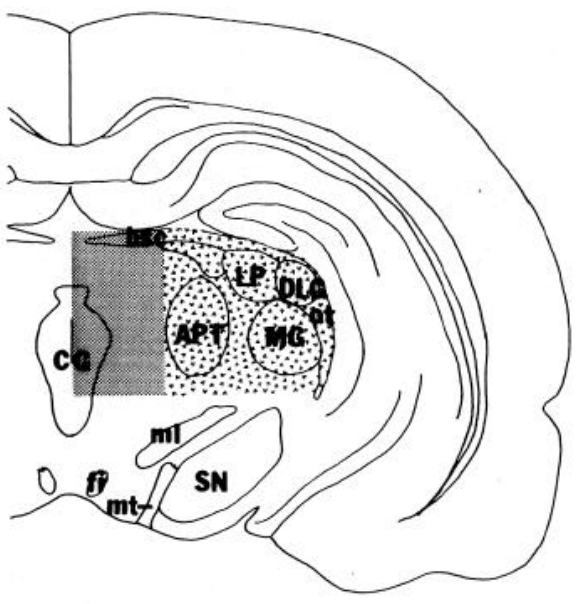

$-5.3 \mathrm{~mm}$

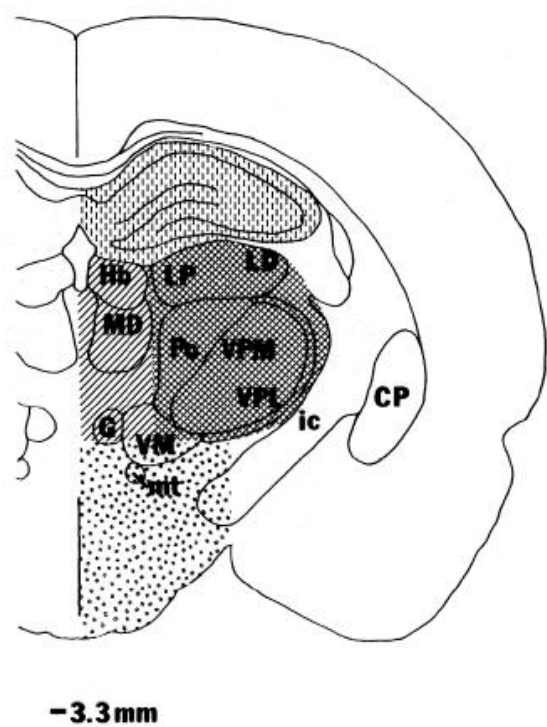

AT-Ant.Thal.

HY-Hypothal.

HF-Hippo.Form.

MT-Med.Thal.

LT-Lat.Thal.

MB-Mam.Bodies

PLT-Post.Lat.Thal.

PMT-Post.Med.Thal.

Figure 1. Brain regions assayed for amino acid concentrations. Schematic diagram indicates the 8 regions of the diencephalon dissected from frozen coronal brain sections and assayed for amino acid levels. Numbers give approximate distance ( $\mathrm{mm}$ ) from bregma based on the atlas of Paxinos and Watson (1986).

$\operatorname{lar}(\mathrm{PF})$ and posterior (Po) thalamic nuclei. Within the rostral intralaminar nuclei (CM, IAM, AM, gelatinosus), the loss of neurons was complete (Fig. $3 B$ ), whereas in the caudal PF and Po nuclei, the loss of neurons was less severe and many surviving neurons were swollen and pale (Fig. $3 D$ ). The mammillary body nuclei were unchanged in 6 animals. In the seventh animal, a small hemorrhage with slight loss of Luxol-stained fibers but no loss of neurons was observed within the medial lateral mammillary body nucleus. In this same animal, small hemorrhages were also present in ventrolateral, anteromedial, medial, and dorsolateral geniculate nuclei of the thalamus. With the exception of an occassional petecchial hemorrhage in medial geniculate nucleus, the brains of all other animals in this group were devoid of hemorrhages.

A careful examination of all available cortical areas, hippocampus, amygdala, hypothalamus, central gray, and midbrain tegmentum revealed no gross microscopic changes.

Late Acute Group 3. Pathological changes were present throughout the diencephalon of this late-stage thiamine-deficient group. The most severe changes were observed within the midline and central regions of the thalamus (Fig. $4 B$ ) and involved complete neuronal loss, vascular proliferation, slight gliosis, spongiform changes, and tissue loss. At rostral thalamus (Fig. $5 B$ ), loss of neurons extended laterally to the ventroposterolateral, ventroposteromedial nuclei, dorsally to the mediodorsal and laterodorsal nuclei, and ventrally to hypothalamus and zona incerta. Hemorrhages and slight to moderate loss of neurons were present within the lateral and subgeniculate nuclei (Fig. $5 B$ ) and medial geniculate nucleus (Figs. $5 E, 6 B$ ). The medial and medial lateral nuclei of the mammillary bodies were also consistently damaged, but unlike other affected areas, the PTD-induced changes consisted almost exclusively of large hemorrhages with no loss of neurons or neuropil in surviving adjacent tissue (Fig. $6 E$ ). The lateral mammillary body nucleus was spared in all but one of the LA animals.

The rostral extent of these lesions was similar to that of the 

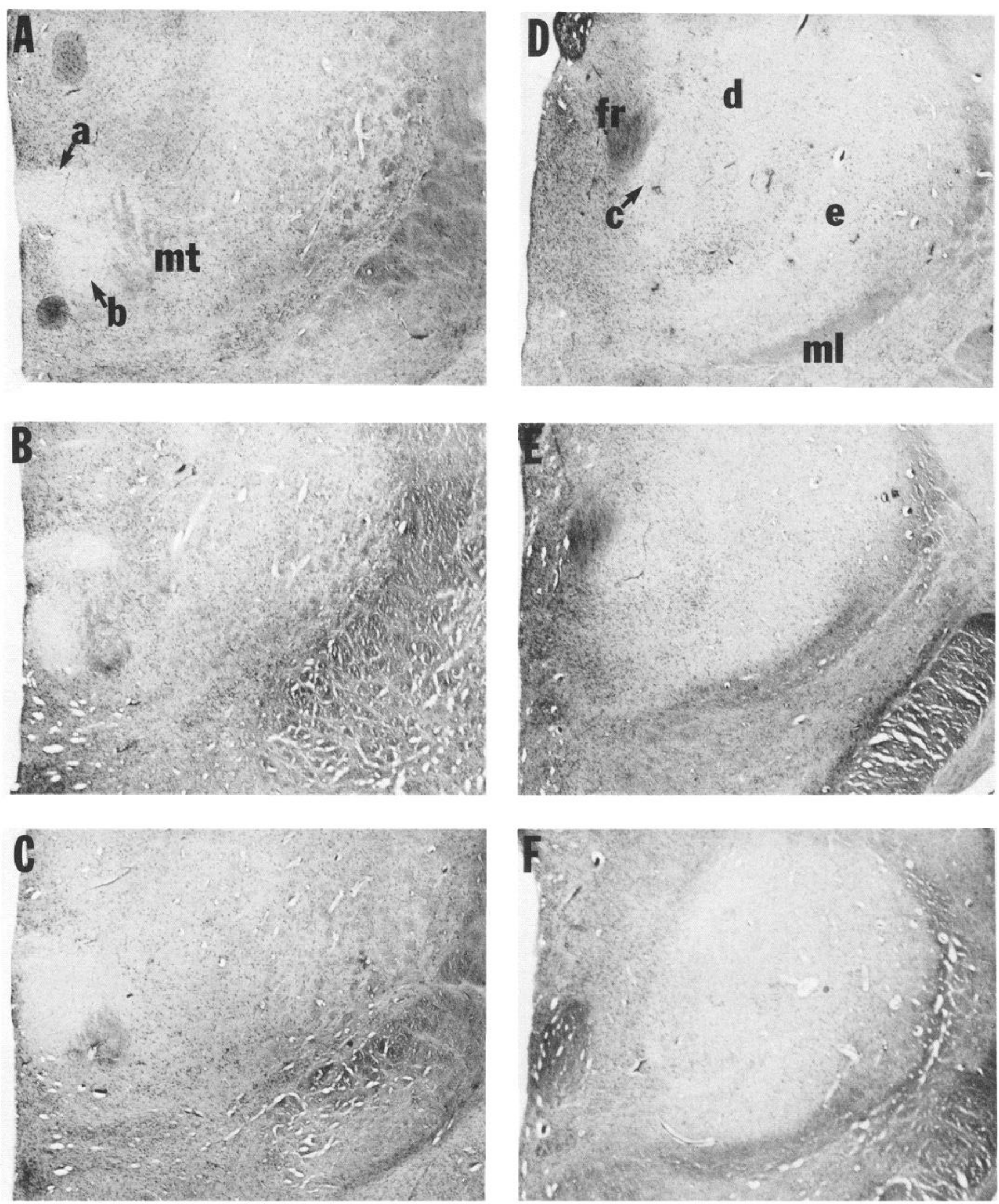

Figure 2. Characteristic appearance and consistency of thalamic lesions in early PTD animals. Luxol fast blue-hematoxylin and eosin-stained coronal sections ( $40 \mu \mathrm{m}$ thick) from 3 cases taken at approximately $2.0 \mathrm{~mm}(A-C)$ and $4.3 \mathrm{~mm}(D-F)$ posterior to bregma. At the anterior thalamus $(A-C)$, loss of neurons and paleness of neuropile are evident within the central medial, interoanteromedial, anteromedial $(a)$, gelatinosus and rhomboid $(b)$ thalamic nuclei. Posteriorly $(D-F)$ these changes are evident in the parafascicular $(c)$, posterior $(d)$, and ventral posteromedial $(e)$ thalamic nuclei. 

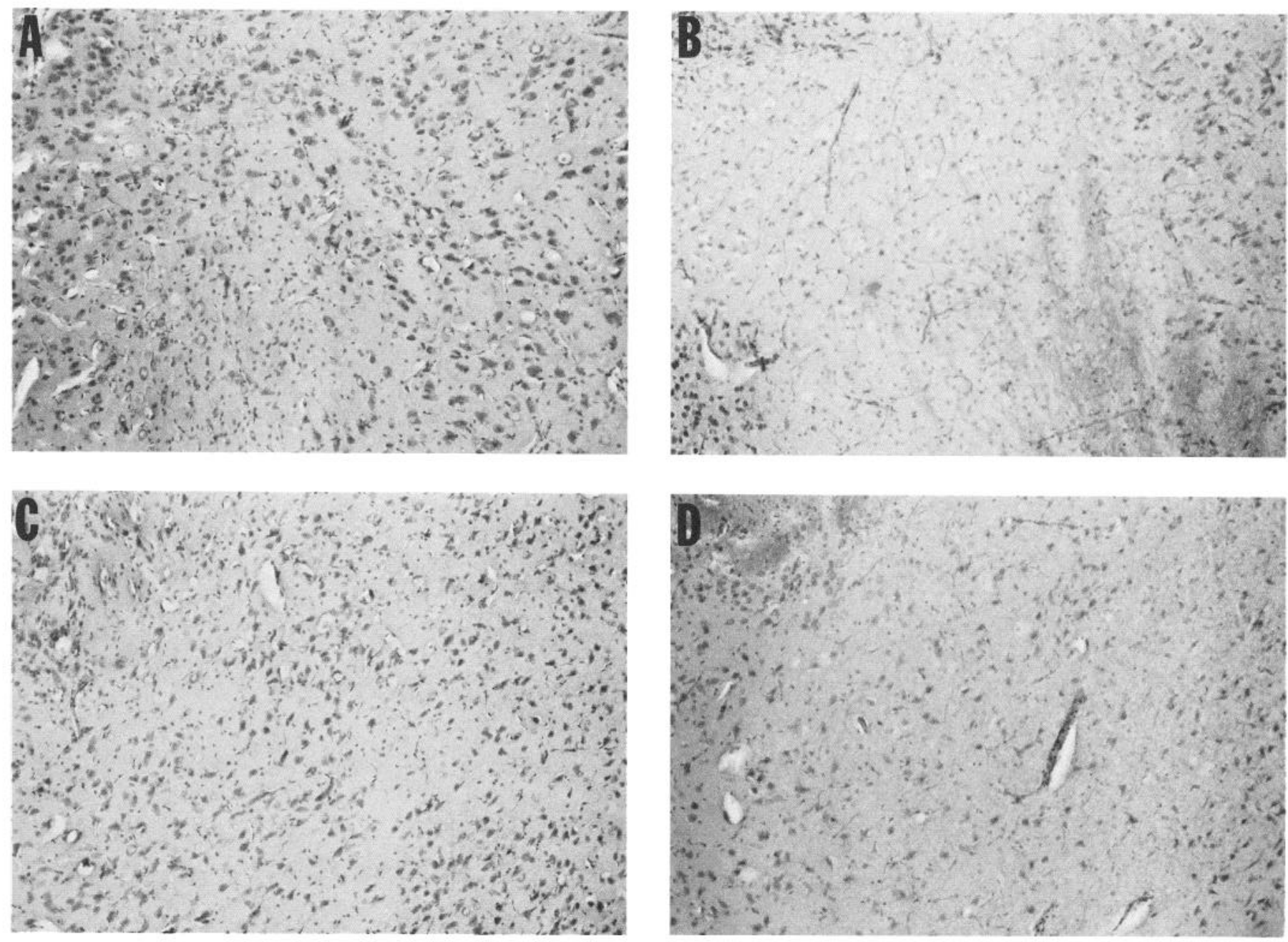

Figure 3. Selective loss of neurons in intralaminar nuclei of early acute PTD cases. Luxol fast blue-hematoxylin and eosin-stained coronal sections (40 $\mu \mathrm{m}$ thick) showing normal CM, gelatinosus $(A)$ and PF, Po $(C)$ thalamic nuclei. In this early acute case killed $1-2 \mathrm{hr}$ following onset of seizures and prior to appearance of opisthotonos, all neurons have disappeared from the anterior $\mathrm{CM}$ and gelatinosus nuclei $(B)$. The remaining cells are glial and endothelial cells. At more posterior level of thalamus, neuronal loss and presence of swollen, pale neurons are evident within the PF and Po thalamic nuclei $(D)$.

early acute group. Caudally, however, cell loss, paleness of neuropile, and small hemorrhages were observed to extend as far as the periacqueductal gray and mesencephalic tegmentum (Fig. $5 E$ ). Hemorrhagic lesions within the linear raphe nucleus (Fig. $5 E$ ) were observed in 5 of the LA animals. No pathologic changes were evident in any other brain structure, including hippocampus, amygdala, and cortex.

Late acute $+M K-801$. A marked reduction in pathologic changes was observed in all of the severely thiamine-deficient animals pretreated with MK-801. Lateral (VPM, VPL), dorsal (MD, AD), and ventral (reuniens, zona incerta) thalamic areas of most animals were relatively spared as evidenced by large numbers of intact neurons, normal staining neuropile, and glial elements (Fig. 5C). A similar preservation of neurons was evident in the mesencephalic tegmental and periacqueductal gray regions (Fig. 5F). Tissue cavitation and spongiform changes were absent in all brains. Furthermore, the number and size of hemorrhages and the severity of vascular changes were markedly reduced within all regions affected in the untreated LA group. This was most evident within the medial geniculate nucleus (Fig. $6 \mathrm{C}$ ) and medial mammillary bodies (Fig. $6 F$ ).

\section{Amino acid concentrations}

\section{PTD treatment}

Acute thiamine deficiency produced significant changes in several of the amino acids measured in thalamus, hypothalamus, mammillary body, and hippocampus (Fig. 7). Aspartate levels were markedly reduced in all brain regions especially within thalamic regions. This decline was greater in the LA than in the EA group. Smaller reductions of glutamate (50-60\% of control) were observed within AT, MT, LT, and PLT regions, whereas glutamate level was increased slightly in the EA group and unchanged in the LA group. Significant increases of glycine were oberved at both stages of PTD in all areas except AT. In rostral regions (MT, LT) glycine levels were higher in the later stage, whereas in more caudal regions (PMT, PLT, HY) glycine levels declined from the early to late stages. In all areas except AT, MT, and LT, GABA content was significantly increased in the EA group but was similar to control in the LA group. Taurine levels were significantly elevated in PMT, PLT, MB, and HY in both EA and LA groups. In a pattern similar to that observed for GABA and glycine, taurine levels in caudal PMT, PLT, and 

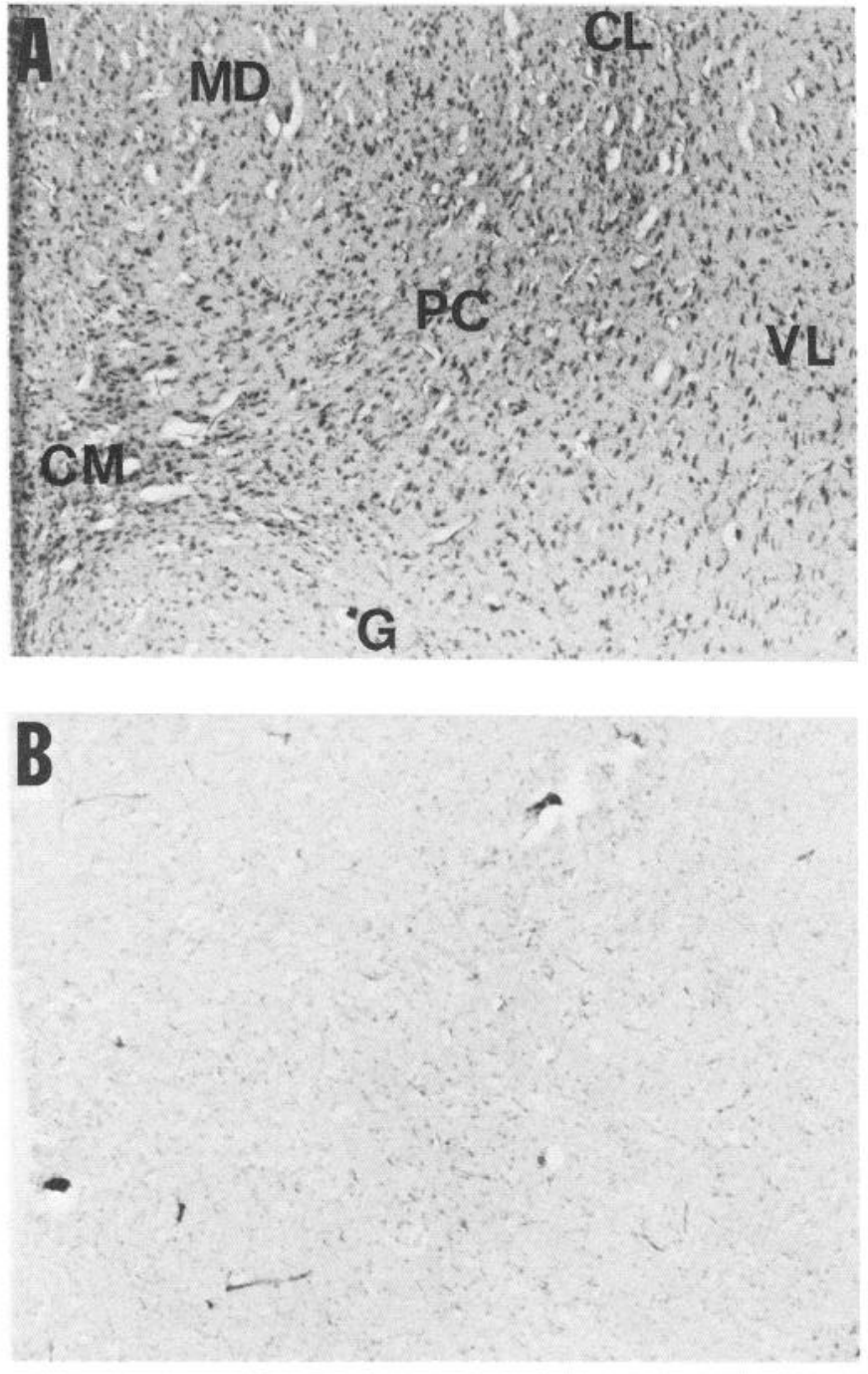

HY regions decreased significantly in the LA compared with EA group.

\section{MK-801 treatment effects-control}

Amino acid levels in the 5 thalamic areas and hippocampus of controls were relatively unchanged by treatment with MK-801 (Table 2). In contrast, HY and MB levels of aspartate, glycine, and GABA were significantly elevated by MK-801 treatment. In addition, a significant decrease of glutamate in HY and an increase of taurine in $\mathrm{MB}$ were observed.

\section{MK-801 treatment effects $-L A P T D$}

Glycine levels in all brain regions were markedly lower in the MK-801 compared with the untreated LA group. Unlike the untreated LA group, glycine levels were similar to control in $\mathrm{MT}, \mathrm{MB}, \mathrm{HY}$, and $\mathrm{HI}$. In all regions except MT and HI, aspartate and glutamate levels were higher but not significantly different from the untreated LA group. Glutamate level in HI of the LA + MK-801 group was significantly lower than both the untreated LA and MK-801 control groups. The levels of GABA in AT, LT, PLT, HY, and MB were significantly lower than the MK-801-treated controls. Taurine levels in LT, PMT, PLT, HY, and MB of the LA + MK-801 group were significantly greater than the MK-801 control group.

Figure 4. Nonhemorrhagic, central lesion of thalamus in LA PTD. $A$, Luxol fast blue-hematoxylin and eosin-stained coronal section $(40 \mu \mathrm{m}$ thick) demonstrating the detail of the midline intralaminar, mediodorsal, and ventrolateral nuclei in a pair-fed control rat. $B$, Corresponding area from the thalamus of a late acute animal killed $18 \mathrm{hr}$ following onset of opisthotonos. Loss of neurons is complete throughout the entire region. The remaining cells are exclusively glial and endothelial cells.

Table 2. Amino acid levels in pair-fed control and MK-801 treated rats

\begin{tabular}{|c|c|c|c|c|c|c|}
\hline \multirow[b]{2}{*}{ Treatment group } & \multirow{2}{*}{$\begin{array}{l}\text { Brain } \\
\text { region }\end{array}$} & \multicolumn{5}{|l|}{ Analyte } \\
\hline & & Aspartate & Glutamate & Glycine & Taurine & GABA \\
\hline Control & AT & $52.6 \pm 2.3$ & $182.6 \pm 9.4$ & $25.3 \pm 1.7$ & $46.7 \pm 3.5$ & $42.0 \pm 4.1$ \\
\hline Control + MK801 & & $50.9 \pm 2.0$ & $203.8 \pm 7.1$ & $30.5 \pm 1.3$ & $52.4 \pm 2.6$ & $70.9 \pm 4.3^{*}$ \\
\hline Control & MT & $43.0 \pm 1.7$ & $170.9 \pm 6.3$ & $18.7 \pm 0.8$ & $31.0 \pm 1.4$ & $22.3 \pm 1.1$ \\
\hline Control + MK801 & & $41.6 \pm 2.0$ & $196.4 \pm 8.0$ & $21.0 \pm 0.7$ & $30.7 \pm 0.9$ & $28.6 \pm 1.4$ \\
\hline Control & LT & $38.5 \pm 2.1$ & $111.9 \pm 4.0$ & $11.3 \pm 0.5$ & $25.3 \pm 1.0$ & $13.2 \pm 0.3$ \\
\hline Control + MK801 & & $32.6 \pm 1.7$ & $116.8 \pm 5.5$ & $13.3 \pm 0.7$ & $23.8 \pm 1.1$ & $14.8 \pm 0.8$ \\
\hline Control & PMT & $45.8 \pm 3.3$ & $128.8 \pm 8.5$ & $25.7 \pm 1.6$ & $27.4 \pm 1.6$ & $42.5 \pm 3.5$ \\
\hline Control + MK801 & & $33.7 \pm 2.3^{*}$ & $110.3 \pm 8.9$ & $24.9 \pm 1.9$ & $22.2 \pm 1.8$ & $40.8 \pm 2.7$ \\
\hline Control & PLT & $68.4 \pm 2.7$ & $134.5 \pm 5.2$ & $18.0 \pm 0.8$ & $27.5 \pm 1.0$ & $25.5 \pm 2.3$ \\
\hline Control + MK801 & & $60.8 \pm 3.0$ & $137.0 \pm 6.7$ & $19.2 \pm 0.6$ & $24.6 \pm 0.8$ & $29.9 \pm 1.6$ \\
\hline Control & HY & $33.6 \pm 2.4$ & $85.4 \pm 6.1$ & $17.3 \pm 1.3$ & $21.0 \pm 1.5$ & $43.2 \pm 2.7$ \\
\hline Control + MK801 & & $48.1 \pm 3.5^{*}$ & $56.1 \pm 2.9^{*}$ & $22.7 \pm 1.3^{*}$ & $22.7 \pm 1.4$ & $71.1 \pm 4.8^{*}$ \\
\hline Control & MB & $38.2 \pm 2.8$ & $106.5 \pm 8.0$ & $19.2 \pm 1.2$ & $19.7 \pm 1.1$ & $45.3 \pm 3.7$ \\
\hline Control + MK801 & & $64.9 \pm 5.0^{*}$ & $138.1 \pm 11.1$ & $28.8 \pm 1.9^{*}$ & $31.2 \pm 2.1^{*}$ & $68.3 \pm 3.8^{*}$ \\
\hline Control & $\mathrm{HI}$ & $32.7 \pm 1.9$ & $126.0 \pm 3.1$ & $18.0 \pm 0.6$ & $77.5 \pm 2.5$ & $22.4 \pm 1.0$ \\
\hline Control + MK801 & & $31.5 \pm 1.1$ & $150.5 \pm 4.9^{*}$ & $21.0 \pm 0.7$ & $79.9 \pm 2.7$ & $24.9 \pm 0.7$ \\
\hline
\end{tabular}

These values represent the concentration (in $\mathrm{nmol} / \mathrm{mg}$ protein) of each amino acid. Values are the averages obtained from 11 animals \pm SEM.

* Significantly different from control ( $p<0.05$, post-hoc Scheffe $F$ test).

Abbreviations: AT, anterior thalamus; HI, hippocampus; HY, hypothalamus; LT, lateral thalamus; MB, mammillary body; MT, medial thalamus; PLT, posterolateral thalamus; PMT, posteromedial thalamus. 

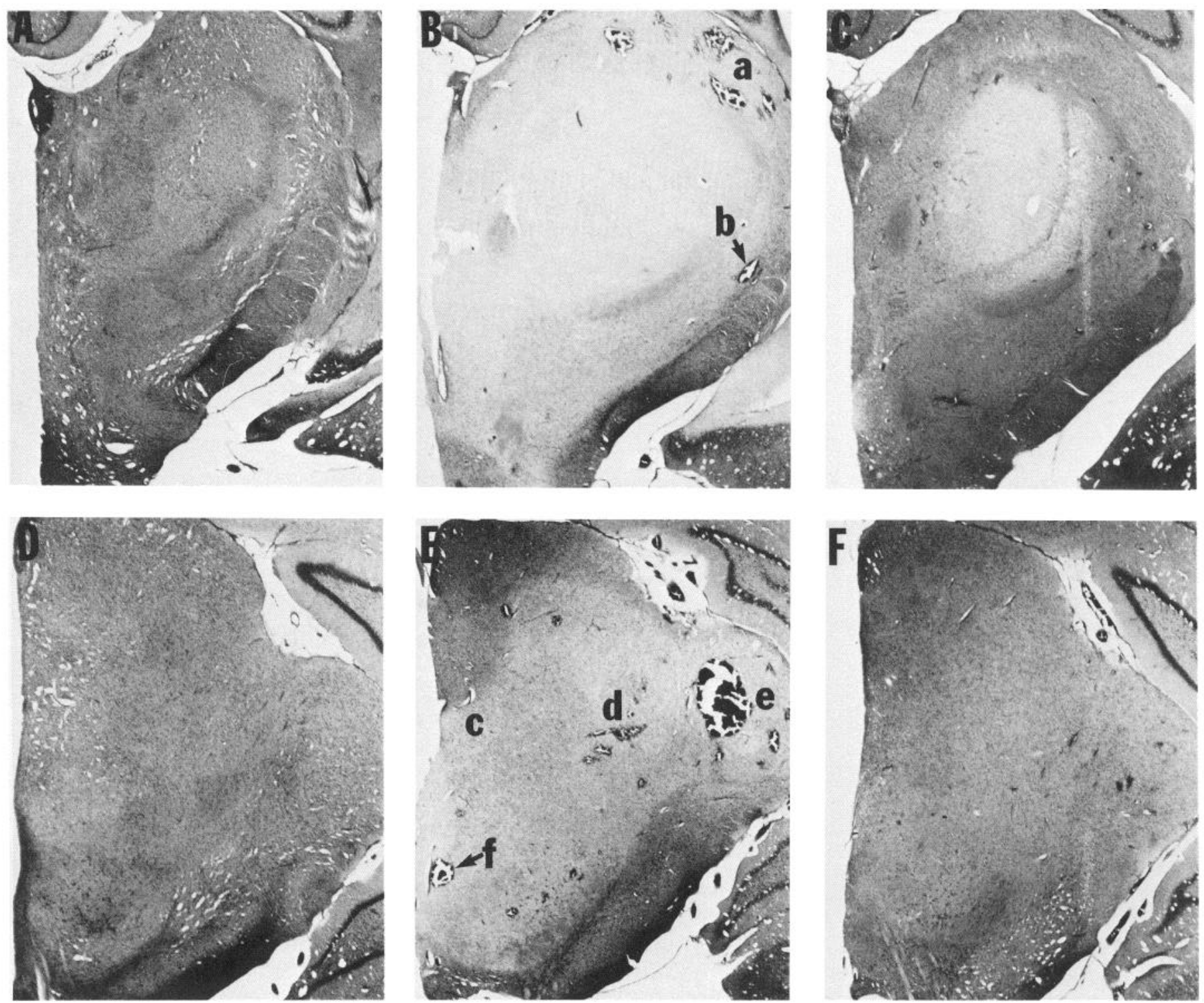

Figure 5. Characteristic appearance of lesions within diencephalic and mesencephalic areas of late acute PTD animals. Luxol fast blue-hematoxylin and eosin-stained coronal sections $(40 \mu \mathrm{m}$ thick) of a pair-fed control case taken at approximately $4.3 \mathrm{~mm}(A)$ and $5.8 \mathrm{~mm}(D)$ posterior to bregma are compared to similar sections taken from late acute $(B, E)$ and late acute + MK-801 $(C, F)$ cases. In the late acute (LA) animal killed $12 \mathrm{hr}$ following onset of opisthotonos, the diencephalic lesion $(B)$ encompases the entire thalamus and extends ventrally into the posterior hypothalamus and laterally to VPM. Loss of tissue is evident in the midline intralaminar region and PF nucleus. Hemorrhages with loss of neurons are present within the lateral geniculate $(a)$ and subgeniculate nuclei $(b)$. In the LA + MK-801 animal $(C)$ killed 12-18 hr after the LA animal, there are only a few small hemorrhages and the loss of neurons is restricted to PF, Po, and the medial part of VPM of the thalamus. Neuronal loss, pallor of neuropil, and occassional hemorrhage within mesencephalic central gray $(c)$, tegmentum $(d)$, medial geniculate $(e)$, and large hemorrhage within linear raphe $(f)$ are evident in the LA case $(E)$, but these changes are virtually absent in the LA + MK-801 case $(F)$.

\section{Discussion}

\section{Pathologic changes}

The highly consistent neuronal loss and paleness of neuropil within CM, IAM, PC, and posterior nuclear group of the EA animals indicate that these are the diencephalic areas most vulnerable to acute thiamine deficiency encephalopathy and the first to develop pathologic changes. The more widespread thalamic damage observed after an additional $24-36 \mathrm{hr}$ of thiamine deficiency (LA group) indicate that as thiamine deficiency becomes more severe, the pathologic process spreads in a radial fashion throughout all areas of thalamus and parts of the hypothalamus. The present findings in the LA group are generally quite similar in both distribution and type of change to those observed in a similarly treated group of PTD rats examined 18 $\mathrm{hr}$ after onset of opisthotonos (Armstrong-James et al., 1988). One important difference is the moderate loss of neurons associated with the hemorrhagic lesions of LP, LG, MG of the present study, which was not observed in an earlier study of late acute PTD brain pathology (Armstrong-James et al., 1988).

In the late acute group, a pattern of pathology was observed that was characterized by selective neuronal loss within intralaminar and posterior nuclear groups and large, multiple hemorrhages with moderate neuronal loss in dorsal-lateral regions. These changes closely resemble the necrotic cell loss within a central zone and hemorrhagic changes with a relative preser- 

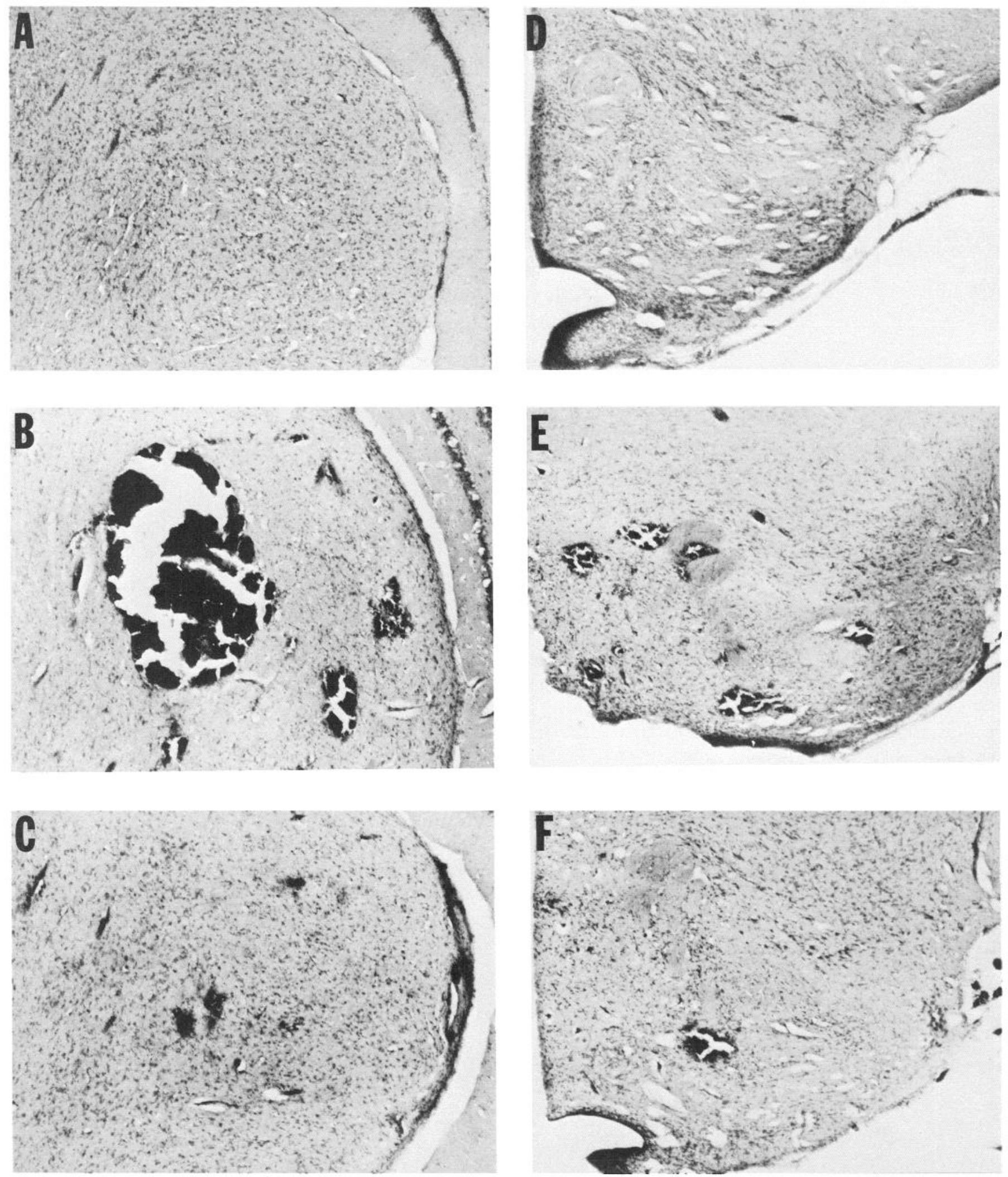

Figure 6. Lesions of the medial geniculate and mammillary body of an LA $(B, E)$ and LA + MK-801 $(C, F)$ PTD animal. $A$, Luxol fast bluehematoxylin and eosin-stained coronal section ( $40 \mu \mathrm{m}$ thick) of the medial geniculate nucleus in a pair-fed control animal. $B$, Corresponding area from a LA animal killed $18 \mathrm{hr}$ following onset of opisthotonos. A large central hemorrhage and several smaller peripheral hemorrhages are present. Moderate to extensive loss of neurons is evident and many of the surviving neurons appear pale and swollen. $C$, Medial geniculate of a LA + MK801 case contains a few very small hemorrhages. Neurons are relatively preserved with an occasional degenerated neuron present in the area adjacent to a hemorrhage. $D$, Stained section of the medial mammillary body in a pair-fed control animal. $E$, corresponding area from the LA case contains several small hemorrhages with little loss of neurons. $F$, Medial mammillary body of the LA + MK-801 case has a single hemorrhage. 
ANTERIOR THALAMUS
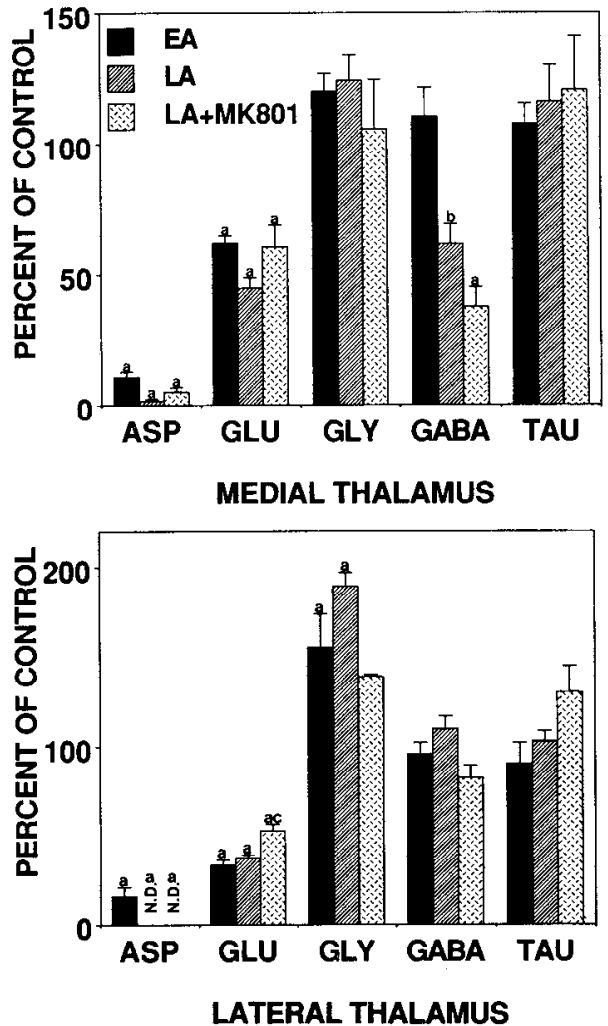

Figure 7. Regional post-mortem brain tissue amino acids of PTD treated groups. Amino acids in the early acute (EA) and late acute (LA) groups are expressed as a percentage of the concentration measured in the pair-fed control group. Amino acid levels of the LA group treated with MK-801 (LA + MK801) are expressed as a percentage of the concentration measured in the pairfed control group administered a similar number of doses of MK-801. Vertical lines indicate SEM. Values are based on the mean of 7 (EA), 10 (LA), and 9 (LA + MK-801) animals. Significant differences (post hoc Scheffe's test, $p<0.05$ ) between EA vs. CT, LA vs. CT, LA + MK-801 vs. CT + MK$801(a)$, LA vs. EA (b), and LA vs. LA + MK-801 (c). Brain regions correspond to those identified in earlier Figure 1. $A S P$, aspartate; $G L U$, glutamate; $G L Y$, glycine; $T A U$, taurine.
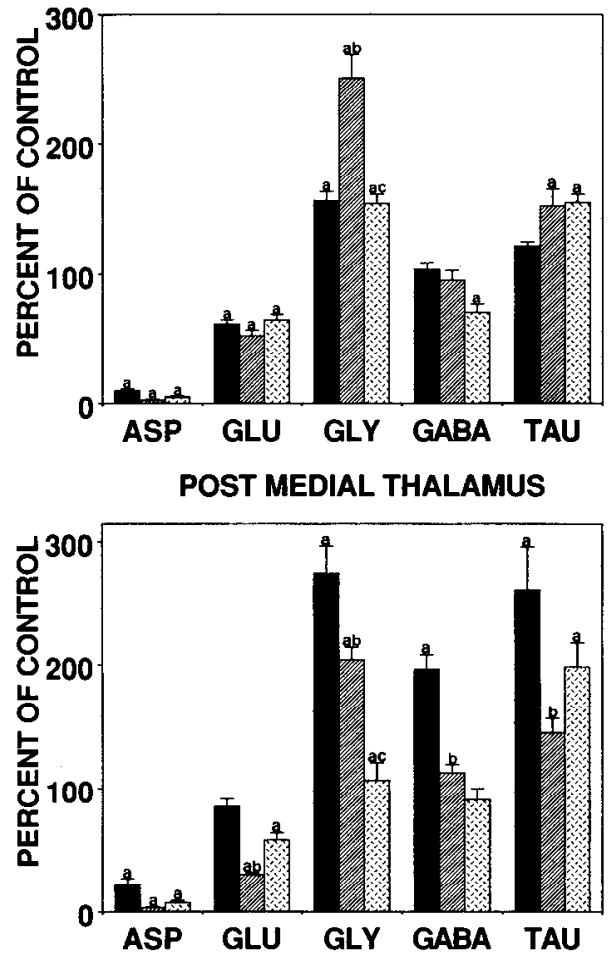

POST LATERAL THALAMUS
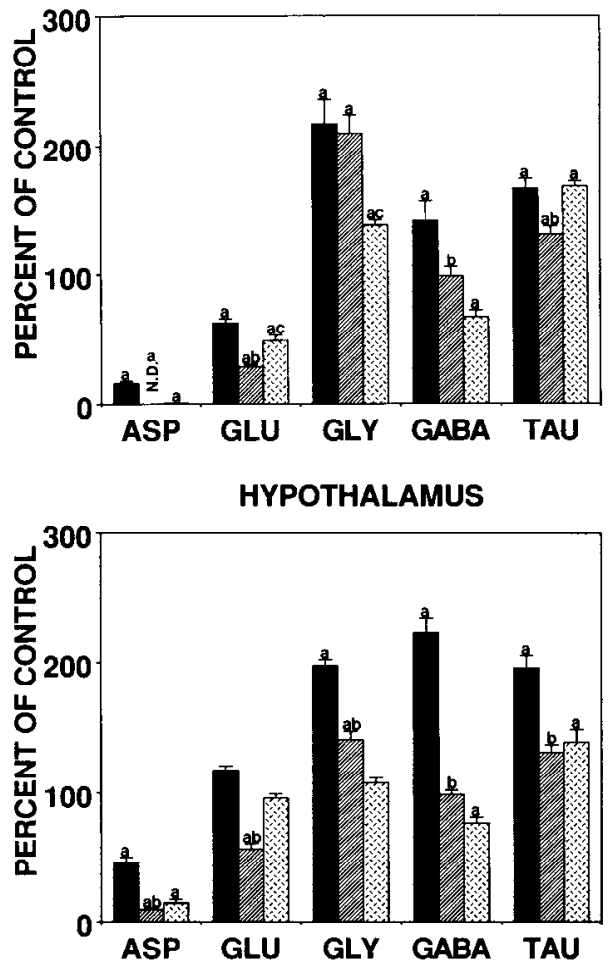

MAMMILLARY BODIES
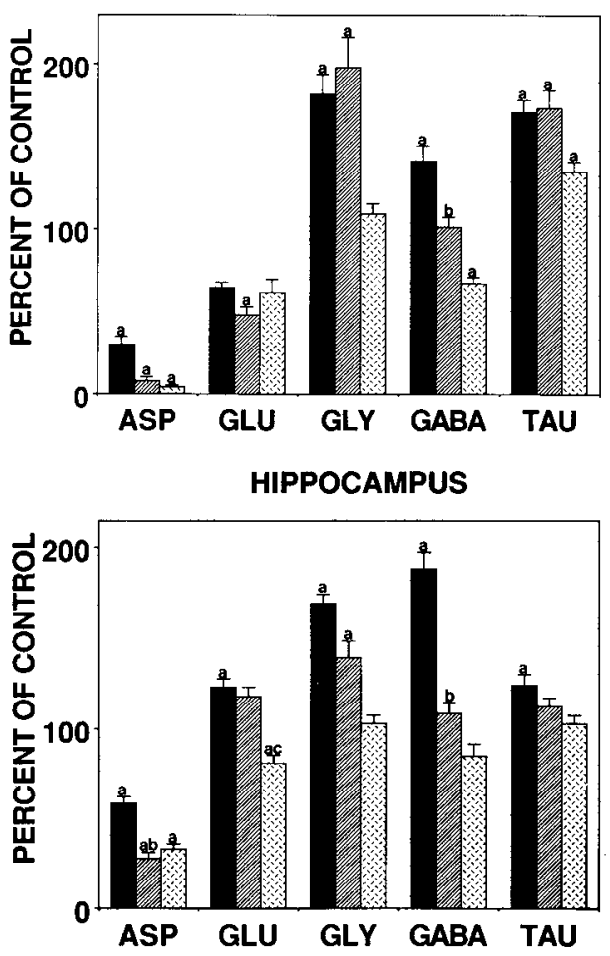

vation of neurons in the peripheral zone of thalamus of PTD rats reported by Papp et al. (1981). The overall distribution of thalamic pathology in the LA group (Fig. $4 B$ ) is strikingly similar to Figure 1 presented in the study of Papp et al. (1981) and suggests that 2 zones of vulnerability to PTD treatment exist within thalamus and diencephalon. The reason for the early and selective nonhemorrhagic, neuronal degeneration within the central core of the thalamus is not known. It has been suggested that the narrow lumen of the penetrating ventral medial branches of the superior cerebellar artery make this region of thalamus highly susceptible to occlusive strokes and nonhemorrhagic infarctions (Rieke, 1987). Swelling of brain parenchyma in the 
late stages of acutc thiaminc deficiency may lead to selective occlusion of these ventral medial arteriolar branches (Armstrong-James et al., 1988). Cessation of blond flow may then result in an ischemic insult to the central regions of the thalamus.

Pathologic damage was markedly attenuated in late-stage PTD animals treated with MK-801. A protective action of MK-801 is supported by the fact that all animals in this group were maintained in acute thiamine deficiency for 12-18 hr longer than the untreated LA group. This drug is a potent noncompetitive, specific NMDA receptor antagonist (Wong et al., 1986) that has been demonstrated effective in blocking ischemic brain lesions (Park et al., 1988) attributed to excessive extracellular levels of excitatory amino acids (Greenamyre, 1986; Rothman and Olney, 1986). The present observations indicate that thiamine deficiency-induced encephalopathy may be treated with NMDA receptor antagonists and that activation of glutamate receptors may be an important pathophysiological mechanism in this disorder. Although MK-801 treatment eliminated seizures and opisthotonos, the possibility that its protective effect is due to nonspecific anticonvulsant actions seems unlikely. Experimentally induced cortical seizures produce lesions within VPL nucleus rather than the intralaminar and posterior thalamic nuclei (Collins and Olney, 1982). Second, urethane administration also eliminates seizures and opisthotonos but provides no protective effect against PTD-induced thalamic lesions (Armstrong-James et al., 1988). Furthermore, entry of $\mathrm{CA}^{2+}$ into neurons is highly correlated with the level of NMDA receptor activation (Rothman and Olney, 1987). The calcium channel blocker nimodipine has been reported to reduce pathophysiological changes in thalamus and mammillary body of symptomatic PTD rat brain (Vogel and Hakim, 1988). These observations suggest that treatment with NMDA receptor antagonists may be effective in preventing brain damage of human WernickeKorsakoff's syndrome.

The mechanism by which overactivation of NMDA receptors could occur selectively within thalamus during acute thiamine deficiency is unknown. The number of NMDA receptors is not a major factor since the hippocampus is totally spared in PTD animals yet has a much higher density of NMDA receptors than any region of thalamus (Greenamyre et al., 1984; Monaghan and Cotman, 1985). Several obervations suggest a sudden rise in extracellular fluid levels of glutamate and aspartate within vulnerable regions could occur during acute thiamine deficiency. Edematous swelling of glia is the earliest morphologic change to occur in thiamine deficiency encephalopathy (Collins, 1967; Robertson et al., 1968; Collins and Converse, 1970; Watanabe and Kanabe, 1978; Watanabe et al., 1981). Glia cells possess a very high uptake rate for aspartate and glutamate (Hertz, 1979) and are considered to play an important role in the rapid inactivation and overall regulation of extracellular levels of excitatory amino acids (Henn, 1976; Walker, 1983; Reynolds and Herschkowitz, 1986). Severe impairment of glia reuptake of amino acids during thiamine deficiency could lead to elevated extracellular levels of glutamate. On the other hand, swelling of glia is a consistent finding in seizure-related, glutamate-induced brain damage (Olney, 1985).

Alternatively, marked hypoperfusion (Hakim, 1986), possibly caused by edematous ischemia of the medial thalamus (Rieke, 1987), occurs during acute thiamine deficiency. Transient ischemia results in an increased release, decreased uptake (Drejer et al., 1985), and highly toxic extracellular levels of excitatory amino acids (Benveniste et al., 1984). Although marked reduc- tions of total aspartate and glutamate levels were observed, there may have been sufficient transmitter levels to produce excitotoxic death of thalamic neurons. Further studies employing in vivo microdialysis measurements will be required to provide a more direct examination of regional changes of these excitatory amino acids before and during the development of pathological changes in PTD rats.

\section{Amino acid changes}

The present study demonstrates a temporal course and regional specificity to previous reports of thiamine deficiency induced amino acid changes (Gaitonde et al., 1975; Butterworth et al., 1979; Hamel et al., 1979; Plaitakis et al., 1979; Butterworth and Heroux, 1989). Marked reductions of total aspartate and glutamate closely paralled the pathological changes. Furthermore, reduced GABA within midbrain-thalamus (Butterworth et al., 1979; Heroux and Buttcrworth, 1988) was found to occur only within the most anterior region of the thalamus and in the LA stages. By contrast, within caudal thalamus, hippocampus, and mammillary bodies, GABA levels increased significantly in the early stages and declined to or below control levels at the later stage. A parallel increase in taurine levels within the same areas of thalamus during the EA stages may indicate a compensatory response of taurine neuromodulatory mechanisms to a sudden overactivation of GABA receptors (Medina and De Robertis, 1984; Malminen and Kontro, 1986).

By contrast, marked increases of glycine (150-275\%) were observed during the late stages of PTD treatment in all brain regions examined. Furthermore, these changes were markedly reduced by pretreatment with $\mathrm{MK}-801$. This effect was most striking in posterior medial and lateral regions of thalamus, areas in which pathologic changes were noticeably attenuated by MK801 .

\section{Appendix}

\section{Abbreviations}

$A M$, anteromedial $\mathrm{n}$. of thalamus; $A P T$, anterior pretectal n.; $A V$, anteroventral $\mathrm{n}$. of thalamus; $b s c$, brachium of superior colliculus; $C G$, central gray; $C L$, centrolateral $\mathrm{n}$. of thalamus; $C M$, centromedial $\mathrm{n}$. of thalamus; $C P$, caudate-putamen n.; $D L G$, dorsolateral geniculate n.; $f$, fornix; $f r$, fasciculus retroflexus; $G$, gelatinosus n. of thalamus; $H b$, habenulum; $i c$, internal capsule; $L D$, laterodorsal $n$. of thalamus; $L H$, lateral hypothalamic area; $L h B$, lateral habenular n.; $L M B$, lateral mammillary body $\mathrm{n}$.; $L P$, lateroposterior $\mathrm{n}$. of thalamus; $M B$, mammillary bodies; $M D$, mediodorsal $\mathrm{n}$. of thalamus; $m f b$, medial forebrain bundle; $M G$, medial geniculate nucleus; $m l$, medial lemniscus; $M M B$, medial mammillary body $n$.; $m t$, mammillothalamic tract; opt, optic tract; $P F$, parafascicular $\mathrm{n}$. of thalamus; $P O$, posterior nuclear group of thalamus; $R E$, reuniens $\mathrm{n}$. of thalamus; $R T$, reticular n. of thalamus; $s m$, stria medullaris; $S M B$, supramammillary body n.; $S N$, substantia nigra n.; $V L$, ventrolateral $\mathrm{n}$. of thalamus; $V M H$, ventromedial hypothalamic area; $V P L$, ventral posterolateral $\mathrm{n}$. of thalamus; and $V P M$, ventral posteromedial $\mathrm{n}$. of thalamus.

\section{References}

Aikawa H, Watanabe IS, Feruse T, Iwasaki Y, Satoyoshi E, Sumi T, Moroji T (1984) Low energy levels in thiamine-deficient encephalopathy. J Neuropathol Exp Neurol 43:276-287.

Armstrong-James M, Ross DT, Chen F, Ebner FF (1988) The effect of thiamine deficiency on the structure and physiology of the rat forebrain. Metab Brain Dis 3:91-124.

Benveniste H, Drejer J, Schousboe A, Diemer NH (1984) Elevation of the extracellular concentrations of glutamate and aspartate in rat hippocampus during transient cerebral ischemia monitored by intracerebral microdialysis. J. Neurochem 43:1369-1374.

Butterworth RF (1986) Cerebral thiamine-dependent enzyme changes 
in experimental Wernicke's encephalopathy. Metab Brain Dis 1:221 228.

Butterworth RF, Heroux M (1989) Effect of pyrithiamine treatment and subsequent thiamine rehabilitation on regional cerebral amino acids and thiamine-dependent enzymes. J Neurochem 52:1079-1084,

Butterworth RF, Hamel E, Landreville F, Barbeau A (1979) Amino acid changes in thiamine-deficient encephalopathy: some implications for the pathogenesis of Fredreich's ataxia. Can J Neurol Sci 6: 217-222.

Collins GH (1967) Glial cell changes in the brain stem of thiamine deficient rats. Am J Pathol 50:791-814.

Collins GH, Converse WK (1970) Cerebellar degeneration in thiamine deficient rats. Am J Pathol 58:219-233.

Collins RC, Olney JW (1982) Focal cortical seizures cause distant thalamic lesions. Science 218:177-179

Drejer J, Beneviste H, Diemer NH, Schousbe A (1985) Cellular origin of ischemic-induced glutamate release from brain tissue in vivo and in vitro. $\mathrm{J}$ Neurochem 45:145-151.

Gaitonde MK, Fayein NA, Johnson AL (1975) Decreased metabolism in vivo of glucose into amino acids of the brain of thiamine-deficient rats. J Neurochem 24:1215-1223.

Gibson GE, Ksiezak-Reding J, Sheu K-FR, Mykytyn V, Blass JP (1984) Correlations of enzymatic, metabolic, and behavioral deficits in thiamin deficiency and its reversal. Neurochem Res 9:803-814.

Greenamyre JT (1986) The role of glutamate in neurotransmission and in neurologic disease. Arch Neurol 16:637-644.

Greenamyre JT, Young AB, Penney JB (1984) Quantitative autoradiographic distribution of $\mathrm{L}-\left[{ }^{3} \mathrm{H}\right]$ glutamate-binding sites in rat central nervous system. J Neurosci 4:2133-2144.

Hakim AM (1984) The induction and reversibility of cerebral acidosis in thiamine deficiency. Ann Neurol 16:673-679.

Hakim AM (1986) Effect of thiamine deficiency and its reversal on cerebral blood flow in the rat. Observations on the phenomena of hyperfusion, "no reflow," and delayed hypoperfusion. J Cereb Blood Flow Metab 6:79-85.

Hakim AM, Pappius HM (1983) Sequence of metabolic, clinical, and histological events in experimental thiamine deficiency. Ann Neurol 13:365-375.

Hamel E, Butterworth RF, Barbeau A (1979) Effect of thiamine deficiency on levels of putative amino acid transmitters in affected regions of the rat brain. J Neurochem 33:575-577.

Henn FA (1976) Neurotransmission and glial cells: a functional relationship? J Neurosci Res 2:271-282

Heroux M, Butterworth RF (1988) Reversible alterations of cerebral $\gamma$-aminobutyric acid in pyrithiamine-treated rats: implications for the pathogenesis of Wernicke's encephalopathy. J Neurochem 51:1221 1226.

Hertz L (1979) Functional interactions between neurons and astrocytes. I. Turnover and metabolism of putative amino acid transmitters. Prog Neurobiol 13:277-289.

Langlais PJ, Mair RG, Anderson CD, McEntee WJ (1988a) Longlasting changes in regional brain amino acids and monoamines in recovered pyrithiamine treated rats. Neurochem Res 13:1199-1206.

Langlais PJ, Mair RG, McEntee WJ (1988b) Acute thiamine deficiency in the rat: brain lesions, amino acid changes and MK-801 pretreatment. Soc Neurosci Abstr 14:774.

Lowry OH, Rosebrough NJ, Farr AL, Randall RJ (1951) Protein measurement with the Folin phenol reagent. J Biol Chem 193:265275.

Mair RG, Anderson CD, Langlais PJ, McEntee WJ (1985) Thiamine deficiency depletes cortical norepinephrine and impairs learning processes in the rat. Brain Res 360:273-284.
Malminen O, Kontro P (1986) Modulation of the GABA-benzodiazepine receptor complex by taurine in rat brain membranes. Neurochem Res 11:85-94.

McCandless DW, Schenker S (1968) Encephalopathy of thiamine deficiency: studies of intracerebral mechanisms. J Clin Invest 47:2268 2280 .

Medina JH, De Robertis E (1984) Taurine modulation of the benzodiazepine- $\gamma$-aminobutyric acid receptor complex in brain membranes. J Neurochem 42:1212-1217.

Monaghan DI, Cotman CW (1985) Distribution of $N$-methyl-D-aspartate-sensitive $\mathrm{L}-\left[{ }^{3} \mathrm{H}\right]$ glutamate-binding sites in rat brain. J Neurosci 5:2909-2919.

Olney JW (1985) Excitatory transmitters and epilepsy-related brain damage. Int Rev Neurobiol 27:337-362.

Papp M, Tarczy M, Takatas A, Auguszt A, Komoly S, Tulok I (1981) Symmetric central thalamic necrosis in experimental thiamine deficient encephalopathy. Acta Neuropathol (Berl) Suppl 8:48-49.

Park CK, Nehls DG, Graham DI, Teasdale GM, McCulloch J (1988) The glutamate antagonist MK-801 reduces focal ischemic brain damage in the rat. Ann Neurol 24:543-551.

Parker WD, Hass R, Stumpf DA, Parks J, Eguren LA, Jackson C (1984) Brain mitochondrial metabolism in experimental thiamine deficiency. Neurology 34:1477-1481.

Paxinos G, Watson C (1986) The rat brain in stereotaxic coordinates, 2nd ed. New York: Academic.

Pincus, JH, Wells K (1972) Regional distribution of thiamine-dependent enzymes in normal and thiamine-deficient brain. Exp Neurol 37:495-501.

Plaitakis A, Nicklas WJ, Berl S (1979) Alterations in uptake and metabolism of aspartate and glutamate in brain of thiamine deficient animals. Brain Res 171:489-502.

Reynolds R, Herschkowitz N (1986) Selective uptake of neuroactive amino acids by both oligodendrocytes and astrocytes in primary dissociated culture: a possible role for oligodendrocyte in neurotransmitter metabolism. Brain Res 371:253-266.

Rieke GK (1987) Thalamic arterial pattern: an endocast and scanning electron microscopic study in normotensive rats. Am J Anat 178:4554.

Robertson DM, Wasan SM, Skinner DB (1968) Ultrastructural features of early brain stem lesions of thiamine-deficient rats. Am J Pathol 52:1081-1097.

Rothman SM, Olney JW (1986) Glutamate and the pathophysiology of hypoxic-ischemic brain damage. Ann Neurol 19:105-111.

Rothman SM, Olney JW (1987) Excitotoxicity and the NMDA receptor. Trends Neurosci 10:299-302.

Thompson SG, McGeer EG (1985) GABA-transaminase and glutamic acid decarboxylase changes in the brain of rats treated with pyrithiamine. Neurochem Res 10:1653-1660.

Vogel S, Hakim AM (1988) Effect of nimodipine on the regiona cerebral acidosis accompanying thiamine deficiency in the rat. J Neurochem 51:1102-1110.

Walker JE (1983) Glutamate, GABA, and CNS disease: a review Neurochem Res 8:521-550.

Watanabe I, Kanabe S (1978) Early edematous lesion of pyrithiamine induced acute thiamine deficient encephalopathy in the mouse. J Neuropathol Exp Neurol 37:401-413.

Watanahe I, Tomita T, Hung K-S, Iwasaki Y (1981) Edematous necrosis in thiamine-deficient encephalopathy of the mouse. J Neuropathol Exp Neurol 40:454-471.

Wong EHF, Kemp JA, Priestley T, Knight AR, Woodruff GN, Iverson LL (1986) The anticonvulsant MK-801 is a potent N-methyl-Daspartate antagonist. Proc Natl Acad Sci USA 83:7104-7108. 